\title{
El proceso estocástico de Feller y el modelo Cox-Ingersoll-Ross: modelación de tasas de interés y valoración de bonos
}

Diego Ismael León Nieto*

* Magíster en Finanzas. Docente-Investigador del Observatorio de Economía y Operaciones Numéricas (ODEON), Universidad Externado de Colombia. Bogotá (Colombia).

[diego.leon@uexternado.edu.co].

Artículo recibido el 25 de octubre de 2017.

Aceptado el 10 de noviembre de 2017.

Para citar este artículo:

León Nieto, D. I. (2017). El proceso estocástico de Feller y el modelo Cox-Ingersoll-Ross: modelación de tasas de interés y valoración de bonos. ODEON, 13, pp. 31-44.

DOI: https://doi.org/10.18601/17941113.n13.03 



\section{Introducción}

La teoría de la modelación de la estructura a término de tasas de interés se basó originalmente en el supuesto de una dinámica unidimensional específica para el proceso instantáneo de la tasa spot $r(t)$. Estos modelos, conocidos como de un factor, implican que el proceso estocástico de tasa corta es suficiente para modelar los cambios en la estructura de plazos de las tasas de interés, es decir, la curva de rendimiento. Esta tasa corta es la tasa instantánea, continuamente compuesta en el tiempo. Para comprender la tasa corta, el canon de mercado más cercano sería una tasa interbancaria overnight. Los modelos de tasa corta de un factor incluyen los de Vasicek (1977), Cox-Ingersoll-Ross (1985), Ho-Lee (1986), Hull-White (1990), Black-Karasinski (1991), Black-Derman-Toy (1990), entre otros.

El padre de todos los modelos anteriores es el de Vasicek (1977), y existen importantes avances a partir de este, con diferentes aportes y variaciones, por ejemplo, en el modelo Ho-Lee existe solo un parámetro de volatilidad relacionado con el proceso de tasa corta, es decir, no presenta un parámetro de reversión a la media. En los modelos Vasicek (1977) y Cox-Ingersoll-Ross (1985) hay dos parámetros relacionados con el proceso de tasa corta, un parámetro de volatilidad y uno de reversión de la media. Elegir estos parámetros para ajustarse mejor a los precios del mercado se denomina calibrar el modelo, ya que estos parámetros de volatilidad y reversión a la media son independientes de la estructura del plazo inicial, y deben determinarse utilizando los precios de mercado de los instrumentos cotizados, como los Caps, los Floors y los Swaps.

En este sentido, un modelo de tasa corta habitualmente se calibra con algunas estructuras iniciales en el mercado, por lo general la curva de rendimiento, la superficie de volatilidad de Caps, la superficie de volatilidad de los Swaptions y, posiblemente, otros productos ya mencionados, determinando así los parámetros del modelo. Vasicek (1977), Cox-Ingersoll-Ross (1985) y Dothan (1978), por ejemplo, se encuentran entre los modelos de tasa corta más utilizados. La fuerza del modelo de Vasicek es que se pueden hallar analíticamente los precios de los bonos y las opciones, sin embargo, las tasas de interés negativas también son posibles con una probabilidad positiva.

Este trabajo centra sus esfuerzos en los hallazgos y aportes del modelo CoxIngersoll-Ross (CIR), ya que surge como una alternativa muy útil a partir de la extensión realizada al modelo Vasicek, puesto que, dados los parámetros es posible obtener tasas de interés positivas con una probabilidad positiva. El documento 
se organiza en cinco partes, la primera está dedicada a la exposición del modelo CIR, luego se expone el proceso estocástico sobre el cual se soporta este modelo, el proceso de Feller (1951); en seguida se analiza la sensibilidad a los parámetros del modelo CIR, luego se presenta el modelo de valoración de bonos y, por último, las conclusiones y recomendaciones del estudio.

\section{Modelo Cox-Ingersoll-Ross (CIR)}

El modelo de Cox-Ingersoll-Ross (1985), que a menudo se cita como CIR, es un modelo continuo de tasa de interés estocástico, conocido como un modelo de tasa de interés de un solo factor. En este, la dinámica instantánea de la tasa de interés viene dada por la siguiente ecuación diferencial estocástica:

$$
d r_{t}=\alpha\left(\beta-r_{t}\right) d t+\sigma \sqrt{r_{t}} d W_{t}
$$

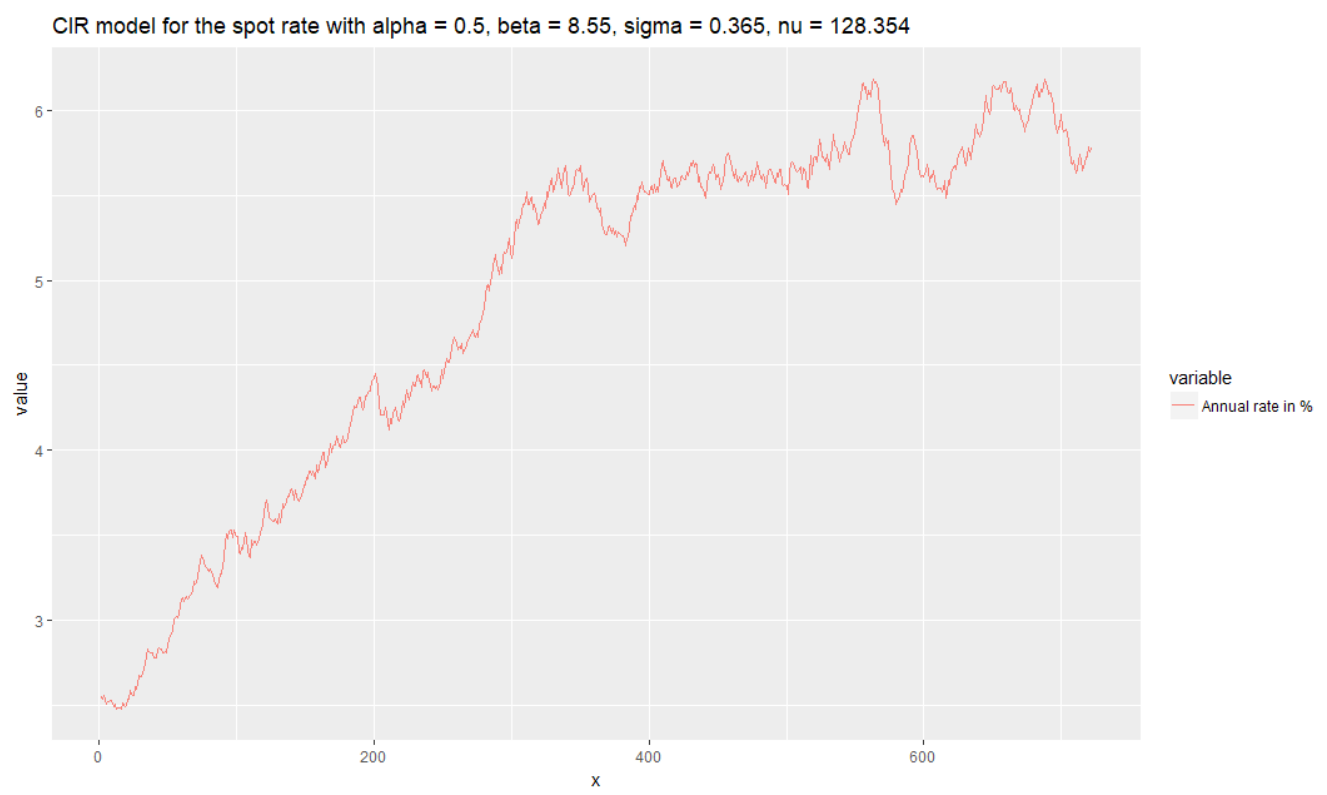

Figura 1. Simulación de un proceso de Feller satisfecho (1)

Aquí, $\alpha, \beta$ у $\sigma$ son constantes positivas, $r_{t}$ es la tasa de interés, $t$ es el tiempo y $W_{t}$ denota el proceso estándar de Wiener; la figura 1 muestra el comportamiento del modelo cIR bajo un proceso de Feller que satisface la ecuación (1) para un va- 
lor de los parámetros definido, $\alpha=0,5$ y $\alpha=36,5 \%$; el valor de largo plazo en la simulación fue determinado como $\beta=8,55 \%$. Es fácil ver que el componente de deriva se comporta igual que en el modelo Vasicek (1977), por tanto, la tasa de interés sigue un proceso de reversión a la media de nuevo; $\beta$ es el promedio de largo plazo, y $\alpha$ es la tasa de ajuste o parámetro de reversión a la media. La diferencia es que el término de volatilidad $\sigma$ no es constante, sino que es proporcional a la raíz cuadrada del nivel de la tasa de interés, $\sigma \sqrt{r_{t}}$. Esta "pequeña" diferencia tiene consecuencias dramáticas con respecto a la distribución de probabilidad de las tasas cortas futuras.

En el modelo CIR, la tasa de interés presenta una distribución chi-cuadrado no central, con la siguiente función de densidad $(f)$ :

$$
f\left[r_{T} \mid r_{t}\right]=2 c \chi_{2 q+2,2 u}^{2}\left[2 c r_{t}\right]
$$

donde:

$$
\begin{gathered}
q=\frac{2 \alpha \beta}{\sigma^{2}}-1, \\
u=c r_{t} e^{-\alpha(T-t)}, \\
y c=\frac{2 \alpha}{\sigma^{2}\left(1-e^{-\alpha(T-t)}\right)} .
\end{gathered}
$$

De esta forma, $\chi_{n, m}^{2}$ denota la función de densidad de probabilidad de la distribución chi-cuadrado, con $n$ grados de libertad, y $m$ indica el parámetro de no centralidad. Como el valor esperado y la varianza de dicha variable aleatoria son $n+m$ y $2(n+2 m)$, respectivamente, tenemos los siguientes momentos para la tasa de interés:

$$
\begin{gathered}
E\left[r_{T} \mid r_{t}\right]=r_{t} e^{-\alpha(T-t)}+\beta\left(1-e^{-\alpha(T-t)}\right) \\
\operatorname{Var}\left[r_{T} \mid r_{t}\right]=\frac{\sigma^{2} r_{t}}{\alpha}\left(e^{-\alpha(T-t)}-e^{-2 \alpha(T-t)}\right)+\frac{\sigma^{2} \beta}{2 \alpha}\left(1-e^{-\alpha(T-t)}\right)^{2}
\end{gathered}
$$


Podemos observar que el valor esperado condicional es exactamente el mismo que en el modelo de Vasicek. Es importante notar que la tasa corta, como una variable normalmente distribuida, puede volverse negativa en el modelo de Vasicek, pero esto no puede ocurrir en el CIR.

Ahora bien, podemos observar cómo los coeficientes determinan la forma de la función de densidad de probabilidad si la trazamos con diferentes conjuntos de parámetros. En la figura 2 se observa que la varianza de la distribución aumenta con el tiempo. $\beta$ cambia la forma de la función de densidad y no solo la desplaza, a diferencia del modelo Vasicek donde el parámetro $\beta$ solo afecta la media de la distribución de probabilidad. Está claro que con un valor mayor de $\alpha$, el proceso alcanza su media a largo plazo antes y tiene menos varianza, y con mayor volatilidad obtenemos una función de densidad más plana, es decir, mayor varianza.
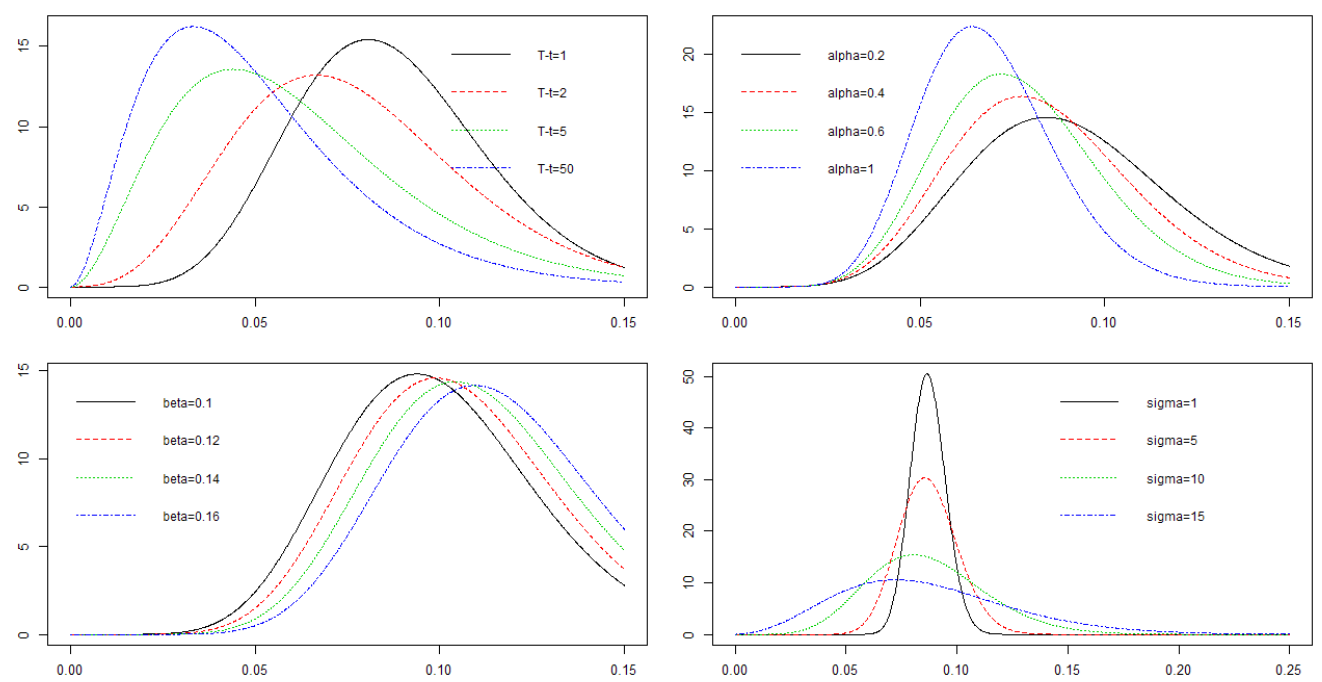

Figura 2. Simulación de funciones de densidad de probabilidad, modelo CIR

\section{Proceso estocástico de Feller y modelo CIR}

Este modelo fue introducido por Feller (1951a; 1951b) para estimar el crecimiento poblacional, y se hizo bastante popular en las finanzas después de que Cox, Ingersoll y Ross lo propusieron para modelar las tasas de interés a corto plazo (Cox et al., 1985). Por su parte Brown y Schaefer (1994), y Andersen y Jesper Lund 
(1997) confirman que el modelo CIR es un buen candidato para evaluar con gran precisión la forma de la estructura a término de las tasas de interés. Recientemente se adoptó para modelar la emisión de óxido nitroso del suelo por Pedersen (2000), y para modelar la tasa de variación evolutiva entre sitios en la evolución molecular (Lepage et al., 2006).

Al contrario del proceso de Ornstein-Uhlenbeck, no hay una solución explícita real para (1). Sin embargo, para algunos valores de $\beta$, se puede construir un proceso estocástico que tenga la misma ley y resolver una ecuación similar. De hecho, para $i \in\{1, \ldots, n\}$, se tiene:

$$
X_{i}(t)=\frac{\sigma}{2} \int_{0}^{t} e^{-\frac{\alpha}{2}(t-s)} d W^{(i)}(s)+e^{-\frac{\alpha}{2} t} \sqrt{\frac{r}{n}},
$$

donde $W^{(i)}, \ldots, W^{(n)}$ son movimientos Brownianos independientes; usando una representación familiar, los procesos $X_{1}, \ldots, X_{n}$ son procesos independientes Ornstein-Uhlenbeck que satisfacen $X_{1}(0)=\sqrt{\frac{r}{n}}, \mathrm{y}$

$$
d X_{i}(t)=-\frac{\alpha}{2} X_{i}(t) d t+\frac{\alpha}{2} d W^{(i)}(t), t>0
$$

De esta forma, se tiene

$$
r_{t}=\sum_{i=1}^{n} X_{i}^{2}(t), t>0
$$

En este sentido, se puede ver que $r(0)=r, \mathrm{y}$

$$
d r(t)=\alpha\left\{n \frac{\sigma^{2}}{4 \alpha}-r(t)\right\} d t+\sigma \sqrt{r(t)} d \hat{W}(t)
$$

donde $\hat{W}$ es un movimiento Browniano definido por 


$$
\hat{W}(t)=\sum_{i=1}^{n} \int_{0}^{t} \frac{X_{i}(s)}{r(s)} d W^{(i)}(s)
$$

Por tanto, (2) es una solución explícita para (1) cuando $\beta=n \frac{\sigma^{2}}{4 \alpha}$, esto es, cuando el parámetro $v$, definido por $v=4 \frac{\alpha \beta}{\sigma^{2}}$, es igual a $n$.

El proceso $r$ definido por (2) no es realmente la solución a (1), ya que la solución no se expresa en términos del movimiento Browniano $W$, sino en términos de un nuevo movimiento Browniano $\hat{W}$. Sin embargo, esto significa que la distribución de ambos procesos es la misma. En Kouritzin y Rémillard (2002) se muestra que se puede construir una solución en un intervalo de tiempo aleatorio bajo la condición $v=\frac{4 \alpha \beta}{\sigma^{2}}=1$.

En Hull y White (1990), donde se expone el modelo conocido como cIR extendido, apuntaban a tener una relación entre la estructura a término de las tasas de interés y los rendimientos sobre los activos libres de riesgo que difieren solo en su tiempo de maduración. La explicación de la estructura del término proporciona información adicional para predecir el efecto sobre la curva de rendimientos si cambiamos las variables subyacentes. La dinámica de tasa corta instantánea corresponde a un proceso autorregresivo de primer orden en tiempo continuo, donde las tasas de interés que se mueven aleatoriamente es elásticamente empujada hacia una ubicación central o un valor de largo plazo, $\beta$, lo cual conduce a la propiedad de reversión a la media.

Si $2 \alpha \beta<\sigma^{2}$, entonces $r_{t}$ puede ser cero. Si $2 \alpha \beta<\sigma^{2}$, entonces el componente de deriva es lo suficientemente grande para que el origen sea inaccesible. Bajo el proceso estocástico que sigue el modelo CIR esta es conocida como la condición de Feller y, en otras palabras, es lo que permite eliminar la posibilidad de tasas de interés negativas.

\section{Sensibilidad a los parámetros del modelo CIR}

En la figura 3 podemos ver que cuanto mayor sea el valor de $\alpha$ que corresponde al parámetro de reversión a la media, la trayectoria alcanza el promedio de largo plazo con mayor velocidad; además, como se ve en las líneas punteadas, ante un mayor nivel de $\alpha$ hay una mayor curvatura en el proceso, lo cual en la práctica puede mostrar un mayor empinamiento en la curva de rendimientos. 
Respecto a la figura 4, a diferencia del modelo Vasicek, el parámetro $\sigma$ no solo amplía la volatilidad a mayor nivel del mismo, sino que cambia la forma del proceso y, por ende, la forma de la curva de rendimientos. Vale la pena observar que el valor esperado converge a $\beta$ cuando $T$ o $\alpha$ van a infinito. Además, la varianza converge a 0 cuando $\alpha$ va al infinito.

10 simulaciones modelo cIR con

$$
\alpha=0,2
$$

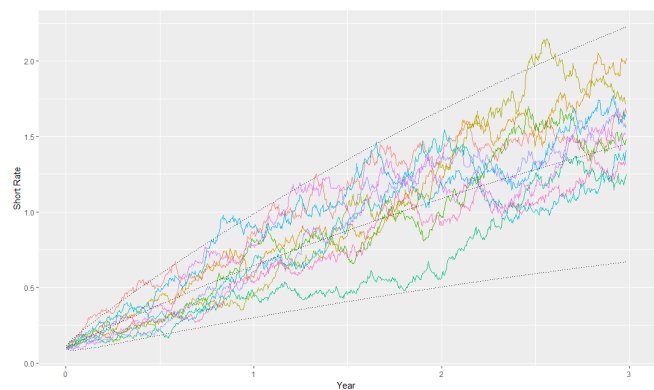

10 simulaciones modelo CIR con

$$
\alpha=0,6
$$

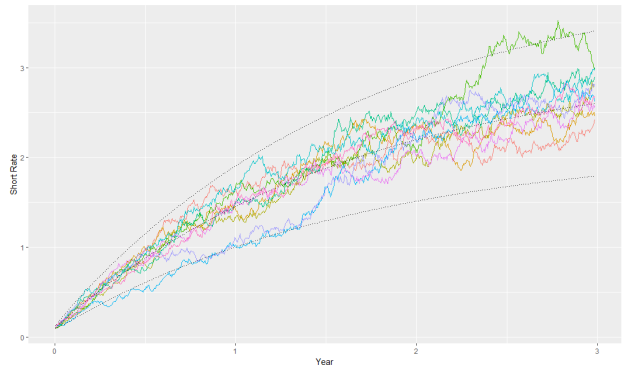

10 simulaciones modelo CIR con

$$
\alpha=0,4
$$

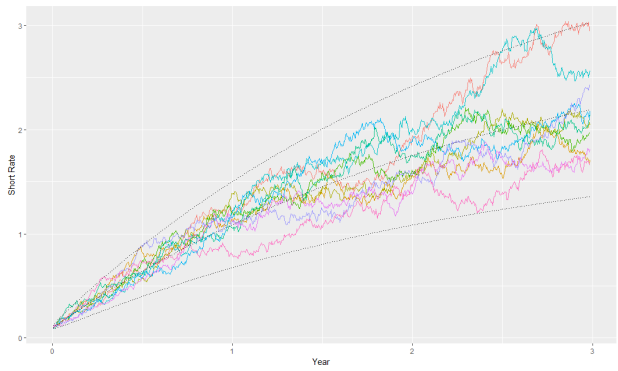

10 simulaciones modelo CIR con

$$
\alpha=0,8
$$

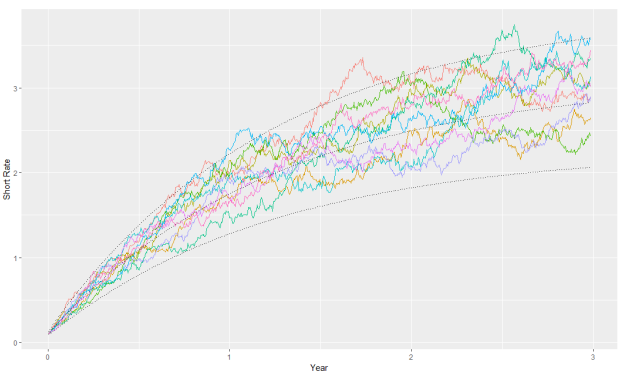

Figura 3. Simulaciones modelo CIR con diferentes valores del parámetro $\alpha$

10 simulaciones modelo cIR con

$$
\sigma=0,2
$$

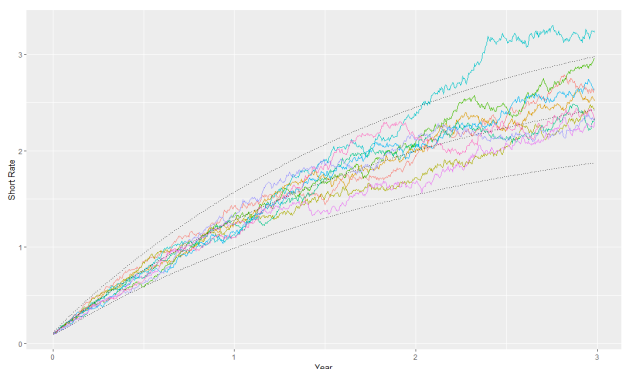

10 simulaciones modelo CIR con

$$
\sigma=0,4
$$

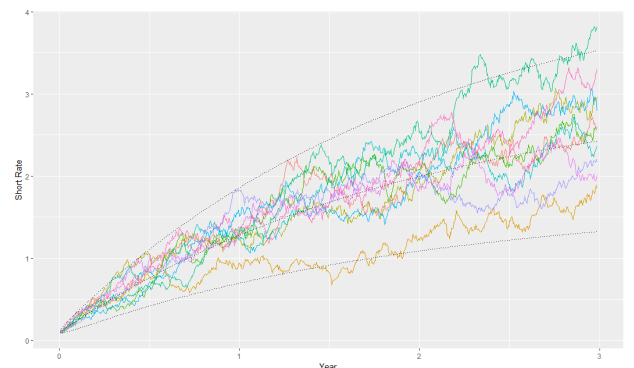

pp. $31-44 \cdot$ n. $^{\circ} 13 / 2017$ 
10 simulaciones modelo cIR con

$$
\sigma=0,6
$$

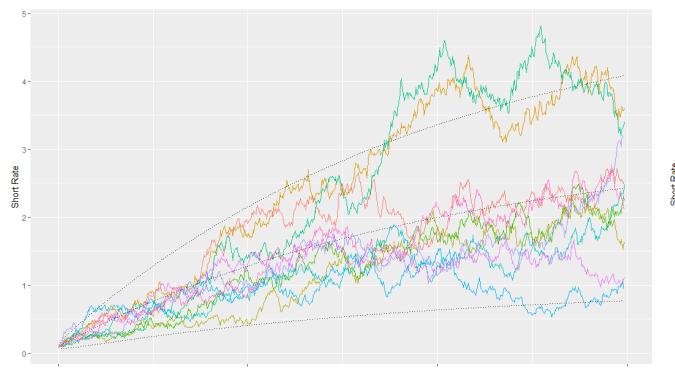

10 simulaciones modelo CIR con

$$
\sigma=0,8
$$

Figura 4. Simulaciones modelo CIR con diferentes valores del parámetro $\sigma$

\section{Valoración de bonos en el modelo cIR}

Supongamos que la tasa de interés spot $r$, expresada como un número, es un proceso de Feller con parámetros $\alpha, \beta, \sigma$ dado por (1). Entonces, un precio de mercado del riesgo de la forma $q(r)=\frac{q_{1}}{\sqrt{r}}+q_{2} \sqrt{r}$, se tiene

$$
P(t, T)=e^{A \tau-\mathrm{r}(t) B \tau}
$$

donde:

$$
\begin{gathered}
\tau=T-t \geq 0, a=\alpha+q_{2} \sigma, b=\frac{\alpha \beta-q_{1} \sigma}{a}, \gamma=\sqrt{a^{2}+2 \sigma^{2}}, \\
B_{\tau}=\frac{2\left(1-e^{-\gamma \tau}\right)}{(\gamma+a)\left(1-e^{-\gamma \tau}\right)+2 \gamma e^{-\gamma \tau}}, \mathrm{y} \\
A_{\tau}=\frac{2\left(1-e^{-\gamma \tau}\right)}{(\gamma+a)\left(1-e^{-\gamma \tau}\right)+2 \gamma e^{-\gamma \tau}}
\end{gathered}
$$

La prueba de este resultado está basada en la fórmula Feynmann-Kac. Para ver el mismo en detalle revisar Lamberton y Lapeyre (2008). 
En la práctica, en lugar del valor del bono es más conveniente trabajar con el correspondiente rendimiento anualizado $R(T, t)$ expresado en porcentaje. Entonces $P(t, T)=e^{-\frac{(T-t) R(t, T)}{100}}$. Utilizando la misma notación para el valor del bono tenemos las funciones $A$ y $B$, de tal forma que la correspondiente tasa spot en porcentaje para una maduración $\tau$, expresada en años es

$$
r(t)=r_{\theta}(t)=\frac{\tau R(t, t+\tau)+100 \times A_{\tau}(\tilde{\theta})}{B_{\tau}(\tilde{\theta})}
$$

donde:

$\theta=\left(\alpha, \beta, \sigma, q_{1}, q_{2}\right)^{T}:$ parámetros bajo el porcentaje de escala

$\tilde{\theta}=\left(\alpha, \beta / 100, \sigma / 10, q_{1} / 10,10 q_{2}\right)^{T}$ : parámetros cuando se utilizan números de punto flotante, esto es, los parámetros sobre los cuales $A_{\tau}$ y $B_{\tau}$ deben ser calculados, como resultado, a diferencia del modelo Vasicek las tasas de largo plazo no dependen de la tasa actual spot.

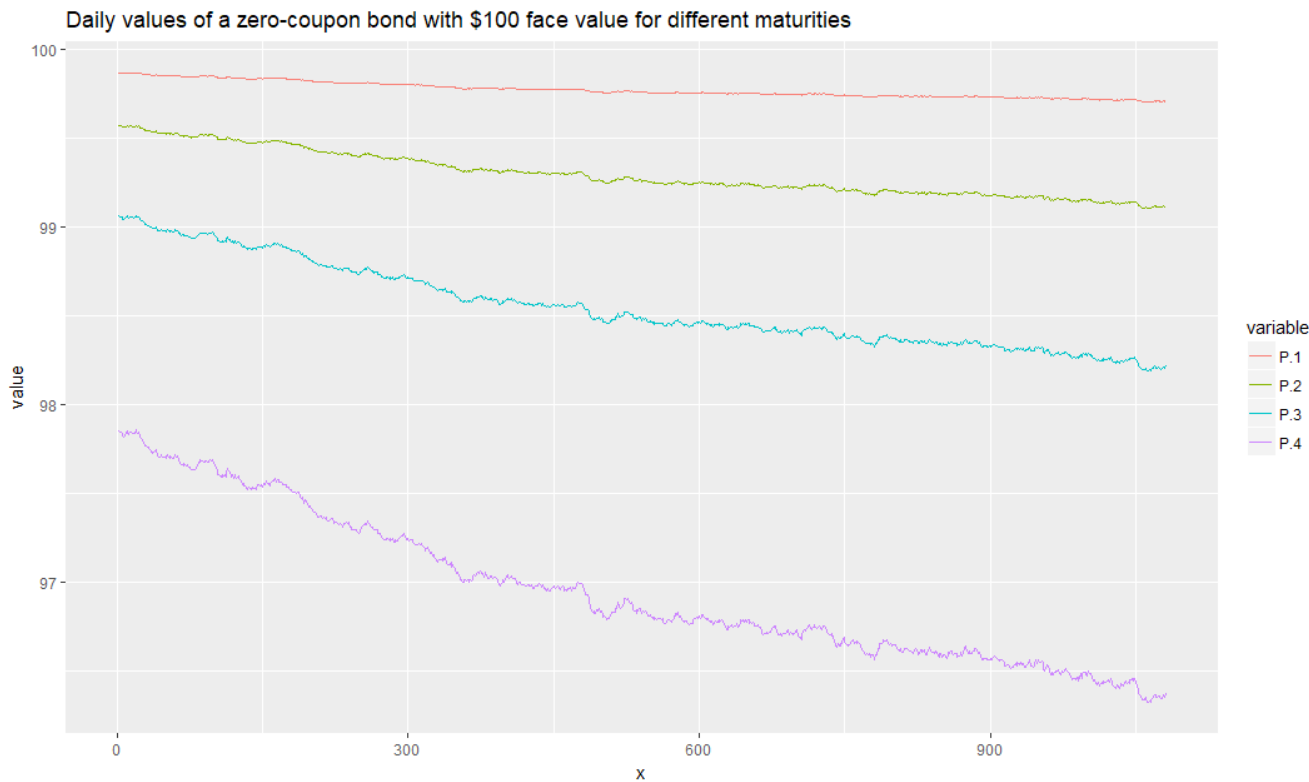

Figura 5. Valores diarios de un bono cero cupón a maduraciones de 1, 3, 6 y 12 meses 


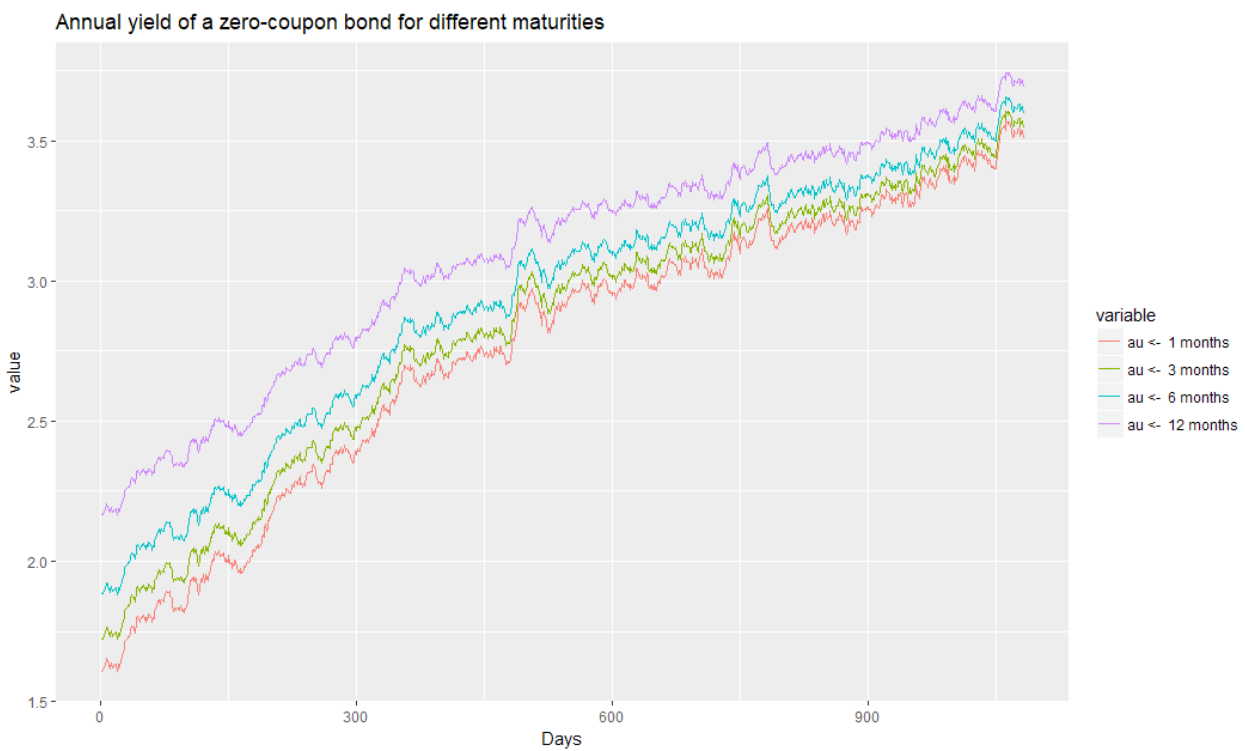

Figura 6. Rendimientos de un bono cero cupón a maduraciones de 1, 3, 6 y 12 meses

En la figura 5 se observa cómo se comporta el valor de un bono cero cupón cuando el proceso de la tasa de interés sigue un modelo cIR; la figura 6 muestra cómo se comportan a diferentes maduraciones los rendimientos del bono cero cupón.

\section{Conclusiones y recomendaciones}

El proceso que sigue la tasa corta en el modelo CIR también se denomina a veces proceso de raíz cuadrada. La buena propiedad de reversión a la media en el modelo de Vasicek se conserva en el modelo CIR. La mala propiedad de la posible negatividad en el modelo de Vasicek se elimina en el modelo de CIR asumiendo $2 \alpha \beta>\sigma 2$ $\mathrm{y}$, por tanto, se asegura que el origen es inaccesible para el proceso, en otros términos, la tasa de largo plazo no depende del nivel de tasa inicial o spot. Por otro lado, la distribución de la tasa corta en el modelo CIR no es normal ni lognormal, pero posee una distribución chi-cuadrado no central.

Dentro de las ventajas que se pueden obtener del modelo CIR se encuentra que no permite tasas de interés negativas, sin embargo, en condiciones como las vividas luego de la crisis financiera de 2008, esta "gran" ventaja se ve relevada por las decisiones tomadas por algunos bancos centrales, como el Banco Central Europeo, de implementar tasas de interés negativas a los depósitos de bancos comerciales en 
las cuentas de alto valor en el banco central. Otra ventaja importante es que la volatilidad condicional depende de la tasa corta, lo cual hace el parámetro de volatilidad susceptible a una calibración más adecuada aunque de manera endógena.

Dentro de las desventajas se pueden encontrar: la baja flexibilidad para ajustar el modelo a una estructura a término observada, se genera una alta correlación entre el valor de los bonos, lo cual empíricamente no es fácil de corroborar; adicionalmente, el modelo no es analíticamente tratable, dado que su solución está basada en herramientas numéricas como el esquema o método de Milstein (1975), sobre el cual se basan los experimentos del presente trabajo.

En torno a posibles extensiones, valdría la pena comparar el modelo CIR con los demás modelos de tasa corta, evaluar su consistencia en términos de parámetros y ajuste a datos observados empíricamente y la capacidad para capturar la dinámica de toda la curva de rendimientos.

\section{Referencias}

Andersen Torben G. Lund, J. (1997). Estimating continous-time stochastic volatility models of the short-term interest rate. Journal of Econometrics, 77, 343-377.

Black, F. y Karasinski, P. (1991). Bond and option pricing when short rates are lognormal. Financial Analysts Journal, 47(4), 52-59.

Black, F., Derman, E. y Toy, W. (1990). A one-factor model of interest rates and its application to Treasury bond options. Financial Analysts Journal, 46, 33-39.

Brown, R. H. Schaefer, S. M (1994). The term structure of real interest rates and the Cox, Ingersoll and Ross Model. Journal of Financial Economics, 35, 3-42.

Cox, J. C., Ingersoll Jr, J. E. y Ross, S. A. (1985). A Theory of the Term Structure of Interest Rates. Econometrica, 53 (2), 385-407.

Dothan L. (1978). On the term structure of interest rates. Journal of Financial Economics, 6, 59-69.

Feller, W. (1951a). Two singular diffusion problems. Annals of Math, 54 (1), 173-182.

Feller, W. (1951b). Diffusion processes in genetics. En Neyman, J. (ed.). Proceedings of the $2^{\text {nd }}$ Berkeley Symposium on Mathematical Statistics and Probability (pp. 227-246). Berkeley: University of California Press. 
Ho, T. y Lee, S. (1986). Term structure movements and pricing interest rate contingent claims. Journal of Finance, 41 (5), 1129-1142.

Hull, J. y White, A. (1990). Pricing Interest Rate Derivative Securities. Review of Financial Studies, 3 (4), 573-592.

Kouritzin, M. A. y Rémillard, B. (2002). Explicit strong solutions of multidimensional stochastic differential equations. Technical report, Laboratory for Research in Probability and Statistics. University of Ottawa-Carleton University.

Lepage, T., Law, S., Tupper, P. y Bryant, D. (2006). Continuous and tractable models for the variation of evolutionary rates. Recuperado de http://arxiv.org/abs/math.PR/0506145.

Lamberton, D. y Lapeyre, B. (2008). Introduction to Stochastic Calculus Applied to Finance (2 edition). Boca Raton, FL: Chapman \& Hall/CRC Financial Mathematics Series,

Milstein, G. N. (1975). Approximate Integration of Stochastic Differential Equations. Theory of Probability \& Its Applications, 19 (3), 557-000.

Pedersen, A.R. (2000). Estimating the nitrous oxide emission rate from the soil surface by means of a diffusion model. Scandinavian Journal of Statistics, 27, 385-403.

Vasicek, O. (1977). An equilibrium characterization of the term structure. Journal of Financial Economics, 5, 177-188. 